\title{
Progression of trunk imbalance in adolescent idiopathic scoliosis with a thoracolumbar/lumbar curve: is it predictable at the initial visit?
}

\author{
Chang Ju Hwang, MD, PhD, Choon Sung Lee, MD, PhD, Dong-Ho Lee, MD, PhD, and \\ Jae Hwan Cho, MD
}

Department of Orthopedic Surgery, Asan Medical Center, University of Ulsan College of Medicine, Seoul, Korea

OBJECTIVE Progression of trunk imbalance is an important finding during follow-up of patients with adolescent idiopathic scoliosis (AIS). Nevertheless, no factors that predict progression of trunk imbalance have been identified. The purpose of this study was to identify parameters that predict progression of trunk imbalance in cases of AIS with a structural thoracolumbar/lumbar (TL/L) curve.

METHODS This study included 105 patients with AIS and a structural TL/L curve who were followed up at an outpatient clinic. Patients with trunk imbalance (trunk shift $\geq 20 \mathrm{~mm}$ ) at the initial visit were excluded. All patients were followed up for more than 2 years. Patients were divided into the following groups according to progression of trunk imbalance: 1) Group P, trunk shift $\geq 20 \mathrm{~mm}$ at the final visit and degree of progression $\geq 10 \mathrm{~mm}$; and 2) Group NP, trunk shift $<20 \mathrm{~mm}$ at the final visit or degree of progression $<10 \mathrm{~mm}$. Radiological parameters included Cobb angle, upper end vertebrae and lower end vertebrae (LEV), LEV tilt, disc wedge angle between LEV and LEV+1, trunk shift, apical vertebral translation, and apical vertebral rotation (AVR). Each parameter was compared between groups. Radiological parameters were assessed at every visit using whole-spine standing anteroposterior radiographs.

RESULTS Among the 105 patients examined, 13 showed trunk imbalance with progression $\geq 10 \mathrm{~mm}$ at the final visit (Group P). Multivariate logistic regression analysis identified a lower Risser grade $(p=0.002)$ and a greater initial AVR $(p=0.020)$ as predictors of progressive trunk imbalance. A change in LEV tilt during follow-up was associated with trunk imbalance $(p=0.001)$.

CONCLUSIONS Risser grade and AVR measured at the initial visit may predict progression of trunk imbalance. Surgeons should consider the risk of progressive trunk imbalance if patients show skeletal immaturity and a greater AVR at the initial visit.

https://thejns.org/doi/abs/10.3171/2017.6.PEDS17313

KEY WORDS adolescent idiopathic scoliosis; trunk imbalance; decompensation; thoracolumbar curve; lumbar curve; spine

A DOLESCENT idiopathic scoliosis (AIS) is a 3D spinal deformity characterized by adolescent onset, coronal curvature, and rotational deformity. Predicting curve progression is critical for determining appropriate timing of bracing or surgery. The magnitude of the curve and skeletal maturity are factors that predict curve progression. ${ }^{3,13}$ Patients with a larger curve and a more immature skeleton are likely to show rapid progression of the curve.

Although predicting curve progression is significant, progression of trunk imbalance is also a crucial finding during follow-up of patients with AIS. Trunk imbalance is defined by a frontal plane shift of the trunk, which is usually a measurement of the horizontal distance between a C-7 plumb line and the central sacral vertical line (CSVL) on whole-spine standing anteroposterior (AP) radiographs. ${ }^{6}$ Trunk imbalance is related to pain and/or the magnitude of the curve and is more common in cases of a thoracolumbar/lumbar (TL/L) curve than in cases of a thoracic curve. ${ }^{6}$ Also, patients with AIS and a TL/L curve

ABBREVIATIONS AIS = adolescent idiopathic scoliosis; $\mathrm{AP}=$ anteroposterior; $\mathrm{AVR}=$ apical vertebral rotation; $\mathrm{AVT}=$ apical vertebral translation; $\mathrm{CSVL}=\mathrm{central}$ sacral vertical line; ICC = intraclass correlation coefficient; LEV = lower end vertebrae; SRS-22 = Scoliosis Research Society-22; TL/L = thoracolumbar/lumbar; UEV = upper end vertebrae.

SUBMITTED June 7, 2017. ACCEPTED June 27, 2017.

INCLUDE WHEN CITING Published online September 8, 2017; DOI: 10.3171/2017.6.PEDS17313. 
require more surgical intervention, even when the curve is $<50^{\circ}$, which is related to trunk shift and low Scoliosis Research Society-22 (SRS-22) scale scores. ${ }^{19}$ Thus, progressive trunk shift in those with a TL/L curve should be monitored more closely.

Several studies have examined the progression of trunk imbalance postoperatively. ${ }^{12,16,21}$ Yet, no study has identified parameters that predict progression of trunk imbalance in patients who do not undergo surgery. Identifying factors that predict progression of trunk imbalance might help to stratify the risk and determine appropriate followup schedules for patients with AIS and a structural TL/L curve. Thus, the aim of this study was to identify parameters that predict progression of trunk imbalance in cases of AIS with a structural TL/L curve.

\section{Methods \\ Study Design}

The electronic medical records and radiographs of patients diagnosed with "adolescent idiopathic scoliosis" at our institution from 2008 to 2013 were retrospectively reviewed. Patients who showed scoliosis with structural lumbar or thoracolumbar curves (Lenke Type 3C, 4C, 5C, or 6C), were 10-19 years old, and were followed up for $\geq$ 2 years were included. The exclusion criteria were other types of AIS, a follow-up period $<2$ years, and surgical treatment before the 2-year follow-up. Finally, 129 patients with AIS and a major TL/L curve were included.

Whole-spine standing AP and lateral radiographs were obtained at the outpatient clinic to evaluate the type and progression of the curve. Regular follow-up was scheduled every 4-6 months or 1 year from the initial visit, depending on the possibility of curve progression. If the curve was $>25^{\circ}$ and the Risser grade was $<2$, then bracing was considered. If the curve was $>50^{\circ}$, then surgical treatment was considered. The study was approved by the IRB of our institution, which waived the requirement for informed consent due to its retrospective nature.

\section{Radiological Evaluation}

AP and lateral plain radiographs were obtained with patients in an upright standing position (both arms were folded forward in the lateral views). Radiological parameters were assessed on whole-spine standing AP radiographs. These parameters included: 1) Cobb angle, the angle between the upper end vertebrae (UEV) and lower end vertebrae (LEV); 2) LEV tilt, the angle between the upper endplate of the LEV and a horizontal line; 3) disc wedging below the LEV, the angle between the lower endplate of the LEV and the upper endplate of the LEV+1; 4) trunk shift, the distance between a C-7 plumb line and a CSVL; 5) apical vertebral translation (AVT), the distance between the center of the apical vertebra and the CSVL; 6) apical vertebral rotation (AVR), the degree of rotation of the apical vertebra according to Nash-Moe grading ${ }^{14}$ and 7) pelvic obliquity. These parameters were measured by 2 independent raters (1 professor who had 5 years of experience in spine surgery [J.H.C.], and 1 fellow). All measurements are illustrated in Fig. 1.

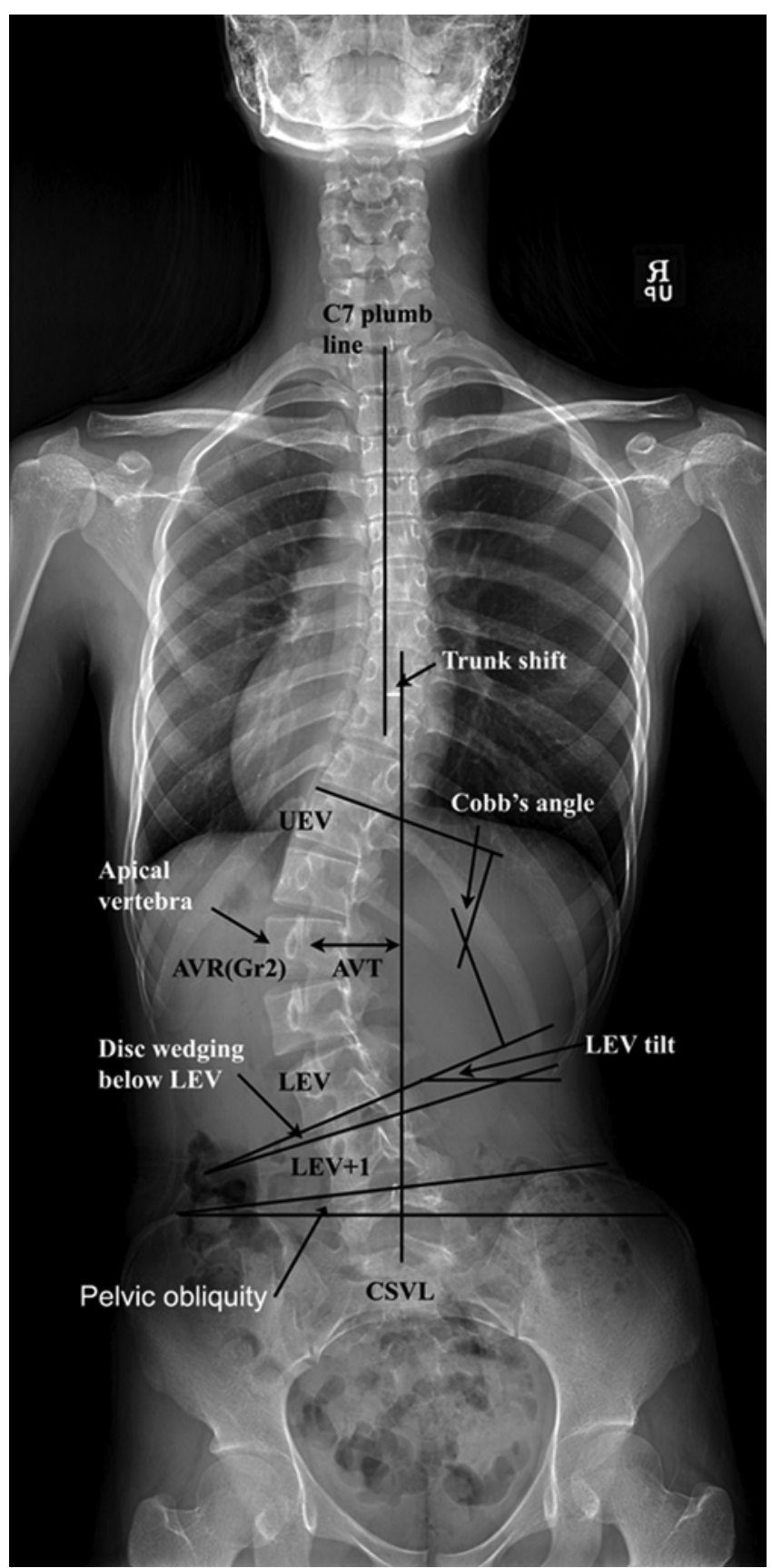

FIG. 1. Measurement of radiological parameters on a standing radiograph. Gr2 = Grade 2 .

\section{Statistical Analyses}

Patients were divided into the following 2 groups according to the progression of trunk shift and the status of trunk balance at the final follow-up: Group P (progression; trunk shift at the final visit $\geq 20 \mathrm{~mm}$ and degree of progression $\geq 10 \mathrm{~mm}$ ) and Group NP (nonprogression; trunk shift at the final visit $<20 \mathrm{~mm}$ or degree of progression $<$ $10 \mathrm{~mm}$ ). Changes in each radiological parameter during the follow-up period were analyzed using the paired t-test or Wilcoxon signed-rank test as appropriate. Comparisons between groups were performed using the independent t- 
test, chi-square test, Fisher's exact test, or Mann-Whitney U-test as appropriate. In addition, variables with a $p$ value $<0.2$ in univariate analysis were entered into multivariate logistic regression analyses, which were conducted using backward stepwise regression. Interobserver reliability was assessed by the intraclass correlation coefficient (ICC) method. All statistical analyses were performed using the Statistical Package for the Social Sciences (version 21.0 , SPSS Inc.); $p$ values $<0.05$ were considered statistically significant.

\section{Results}

Among the 129 patients with AIS and a TL/L curve, 24 showing trunk imbalance $(>20 \mathrm{~mm})$ at the initial visit were excluded. A total of 105 patients (15 boys and 90 girls) were included in the study. The mean age was 14.4 \pm 2.8 years, the average Risser grade at the initial visit was $2.1 \pm 1.8$, and the average follow-up period was 3.6 \pm 1.4 years. Surgery was performed in 76 patients. T-11 was the most common UEV ( $\mathrm{n}=32,30.5 \%)$, followed by T-6 $(\mathrm{n}=22,21.0 \%), \mathrm{T}-12(\mathrm{n}=12,11.4 \%)$, and T-7 $(\mathrm{n}=11$, $10.5 \%)$. L-4 was the most common LEV $(\mathrm{n}=32,30.5 \%)$, followed by L-3 $(\mathrm{n}=20,19.0 \%), \mathrm{T}-12(\mathrm{n}=19,18.1 \%)$, and T-11 $(n=14,13.3 \%)$. Mean values of the degree of curve, LEV tilt, disc wedging below the LEV, AVT, AVR, and pelvic obliquity all significantly deteriorated during follow-up (Table 1). However, the degree of trunk shift did not change over time $(\mathrm{p}=0.970)$.

Among the 105 patients enrolled in the study, 14 showed trunk imbalance at the final visit. However, only 13 belonged to Group P (the exception was a patient who showed progression of trunk shift $<10 \mathrm{~mm}$ ). Univariate analyses identified younger age (13.1 vs 14.5 years, $\mathrm{p}=$ 0.107 ), a lower Risser grade (1.0 vs $2.2, \mathrm{p}=0.020)$, a greater AVR (1.4 vs $1.1, p=0.175)$, and smaller pelvic obliquity ( 0.8 vs $1.4, p=0.196)$ as factors related to progressive trunk imbalance (Group P). However, multivariate logistic regression analysis identified a lower Risser grade $(\mathrm{p}=$ $0.009)$ and a greater AVR ( $=0.031)$ as being associated with progression of trunk imbalance (Table 2).

The relationship between changes in each radiological parameter and progression of trunk imbalance is shown in Table 3. A change in LEV tilt was the only parameter significantly related to progression of trunk imbalance $(\mathrm{p}$ $=0.001$ ). While the LEV tilt increased by $5.9^{\circ}$ (from $15.8^{\circ}$

TABLE 1. Comparison of radiological parameters between the initial examination and final follow-up

\begin{tabular}{lccr}
\hline \multicolumn{1}{c}{ Radiological Parameter } & Initial Visit & Final Visit & p Value \\
\hline Cobb angle $\left(^{\circ}\right)$ & $32.7 \pm 10.3$ & $45.0 \pm 14.2$ & $<0.001$ \\
\hline LEV tilt $\left(^{\circ}\right)$ & $15.9 \pm 5.8$ & $22.8 \pm 7.9$ & $<0.001$ \\
\hline Disc wedging below LEV $\left(^{\circ}\right)$ & $1.9 \pm 2.3$ & $2.9 \pm 3.4$ & 0.004 \\
\hline Trunk shift $(\mathrm{mm})$ & $2.2 \pm 10.1$ & $2.2 \pm 12.9$ & 0.970 \\
\hline AVT $(\mathrm{mm})$ & $25.8 \pm 11.9$ & $38.5 \pm 14.3$ & $<0.001$ \\
\hline AVR $($ grade) & \\
\hline Pelvic obliquity $\left(^{\circ}\right)$ & $1.1 \pm 0.7$ & $1.8 \pm 0.7$ & $<0.001$ \\
\hline Aldag & $1.1 \pm 1.4$ & $1.4 \pm 1.5$ & 0.003 \\
\hline
\end{tabular}

All data given as mean $\pm S D$, unless otherwise indicated.

${ }^{*}$ Wilcoxon signed-rank test was used.
TABLE 2. Comparison of parameters between Group NP and Group $P$ at the initial visit

\begin{tabular}{|c|c|c|c|c|}
\hline \multirow[b]{2}{*}{ Parameter } & \multirow{2}{*}{$\begin{array}{l}\text { Group } \\
\text { NP }\end{array}$} & \multirow{2}{*}{$\begin{array}{l}\text { Group } \\
\text { P }\end{array}$} & \multicolumn{2}{|c|}{$p$ Value } \\
\hline & & & Univariate & Multivariate \\
\hline No. of patients & 92 & 13 & & \\
\hline Age (yrs) & $14.5 \pm 3.0$ & $13.1 \pm 2.7$ & 0.107 & 0.826 \\
\hline $\operatorname{Sex}(M: F)$ & $12: 80$ & $3: 10$ & 0.393 & NE \\
\hline Risser grade* & $2.2 \pm 1.8$ & $1.0 \pm 1.7$ & 0.020 & 0.009 \\
\hline $\begin{array}{l}\text { Follow-up period } \\
\text { (yrs) }\end{array}$ & $3.6 \pm 1.5$ & $3.7 \pm 1.4$ & 0.773 & NE \\
\hline Cobb angle $\left({ }^{\circ}\right)$ & $32.4 \pm 10.4$ & $35.5 \pm 9.0$ & 0.312 & NE \\
\hline LEV tilt $\left({ }^{\circ}\right)$ & $15.8 \pm 5.9$ & $16.5 \pm 4.9$ & 0.648 & NE \\
\hline $\begin{array}{l}\text { Disc wedging } \\
\text { below LEV }\left(^{\circ}\right)\end{array}$ & $1.9 \pm 2.3$ & $2.2 \pm 2.3$ & 0.602 & NE \\
\hline Trunk shift (mm) & $2.1 \pm 10.2$ & $2.8 \pm 9.8$ & 0.838 & $\mathrm{NE}$ \\
\hline $\mathrm{AVT}(\mathrm{mm})$ & $25.5 \pm 11.9$ & $27.6 \pm 11.8$ & 0.553 & NE \\
\hline AVR (grade) ${ }^{*}$ & $1.1 \pm 0.7$ & $1.4 \pm 0.9$ & 0.175 & 0.031 \\
\hline Pelvic obliquity $\left({ }^{\circ}\right)$ & $1.4 \pm 1.8$ & $0.8 \pm 1.0$ & 0.196 & 0.075 \\
\hline
\end{tabular}

$\mathrm{NE}=$ not entered

All data given as mean $\pm S D$, unless otherwise indicated.

* Mann-Whitney U-tests were used.

to $21.7^{\circ}$ ) in Group NP, it increased by $13.8^{\circ}$ (from $16.5^{\circ}$ to $30.3^{\circ}$ ) in Group P. At final follow-up, all radiological parameters (except AVT and pelvic obliquity) in Group $\mathrm{P}$ showed a more severe degree than those in Group NP (Table 4). An illustrative case is presented in Fig. 2.

Interobserver agreements (ICCs) were strong for measurements of the degree of curves (0.943), LEV tilt (0.916), degree of trunk shift (0.889), AVT (0.979), AVR (0.810), and pelvic obliquity (0.946). However, interobserver agreements for measurements of disc wedging below the LEV were moderate $(\mathrm{ICC}=0.601, \mathrm{p}=0.013)$.

\section{Discussion}

In this study cohort, many patients who required surgery before the 2-year follow-up were excluded. The main reason for the early surgery was a severe degree of scoliosis at the initial visit, or rapid progression of the curve without reference to progression in truncal imbalance.

TABLE 3. Changes in radiological parameters at follow-up

\begin{tabular}{lccr}
\hline Radiological Parameter Change & Group NP & Group P & p Value \\
\hline Cobb angle $\left(^{\circ}\right)$ & $12.4 \pm 14.7$ & $19.5 \pm 12.6$ & 0.104 \\
\hline LEV tilt $\left(^{\circ}\right)$ & $5.9 \pm 8.0$ & $13.8 \pm 8.3$ & 0.001 \\
\hline Disc wedging below LEV $\left(^{\circ}\right)$ & $0.7 \pm 3.0$ & $2.8 \pm 5.7$ & 0.228 \\
\hline Trunk shift $(\mathrm{mm})$ & $7.7 \pm 6.3$ & $17.9 \pm 8.0$ & $<0.001$ \\
\hline AVT $(\mathrm{mm})$ & $16.7 \pm 15.5$ & $27.5 \pm 26.5$ & 0.176 \\
\hline AVR (grade) $\dagger$ & $0.7 \pm 0.8$ & $0.9 \pm 0.9$ & 0.470 \\
\hline Pelvic obliquity $\left(^{\circ}\right)$ & $0.3 \pm 1.1$ & $0.5 \pm 0.8$ & 0.634 \\
\hline
\end{tabular}

All data given as mean $\pm S D$, unless otherwise indicated.

* Changes in each parameter between the initial and final visits.

† Mann-Whitney U-tests was used. 
TABLE 4. Comparison of parameters between Group NP and Group $P$ at the final visit

\begin{tabular}{lccr}
\hline \multicolumn{1}{c}{ Parameter } & Group NP & Group P & p Value \\
\hline Cobb angle $\left(^{\circ}\right)$ & $44.8 \pm 14.1$ & $54.9 \pm 11.3$ & 0.015 \\
\hline LEV tilt $\left(^{\circ}\right)$ & $21.7 \pm 7.4$ & $30.3 \pm 6.6$ & $<0.001$ \\
\hline Disc wedging below LEV $\left(^{\circ}\right)$ & $2.6 \pm 3.0$ & $5.0 \pm 5.3$ & 0.017 \\
\hline Trunk shift $(\mathrm{mm})$ & $8.6 \pm 5.2$ & $25.5 \pm 3.1$ & $<0.001$ \\
\hline AVT $(\mathrm{mm})$ & $38.0 \pm 13.8$ & $42.4 \pm 17.8$ & 0.299 \\
\hline AVR $(\text { grade })^{*}$ & $1.7 \pm 0.6$ & $2.2 \pm 0.7$ & 0.011 \\
\hline Pelvic obliquity $\left(^{\circ}\right)$ & $1.4 \pm 1.5$ & $1.3 \pm 1.3$ & 0.792 \\
\hline
\end{tabular}

All data given as mean $\pm \mathrm{SD}$, unless otherwise indicated.

* Mann-Whitney U-test was used.
However, a few patients showed progressive truncal imbalance, which was the reason for the surgery.

In general, patients with AIS and a TL/L curve complain of greater discomfort than those with a thoracic curve, even though the former may be smaller. ${ }^{4}$ This may be due to trunk imbalance, which is related to pain and/ or functional disability. ${ }^{7}$ Thus, predicting trunk imbalance is important for patients with AIS and a structural TL/L curve (Lenke Type 3C, 4C, 5C, or 6C). In addition, trunk imbalance affects progression of a scoliotic curve. ${ }^{8}$ Furthermore, the trunk shift at final follow-up is related to the magnitude of the curve at the initial visit, although the correlation is weak $(r=0.32) .{ }^{6}$ However, the results of the present study do not support this. We found no difference in the initial Cobb angle and the degree of progression be-

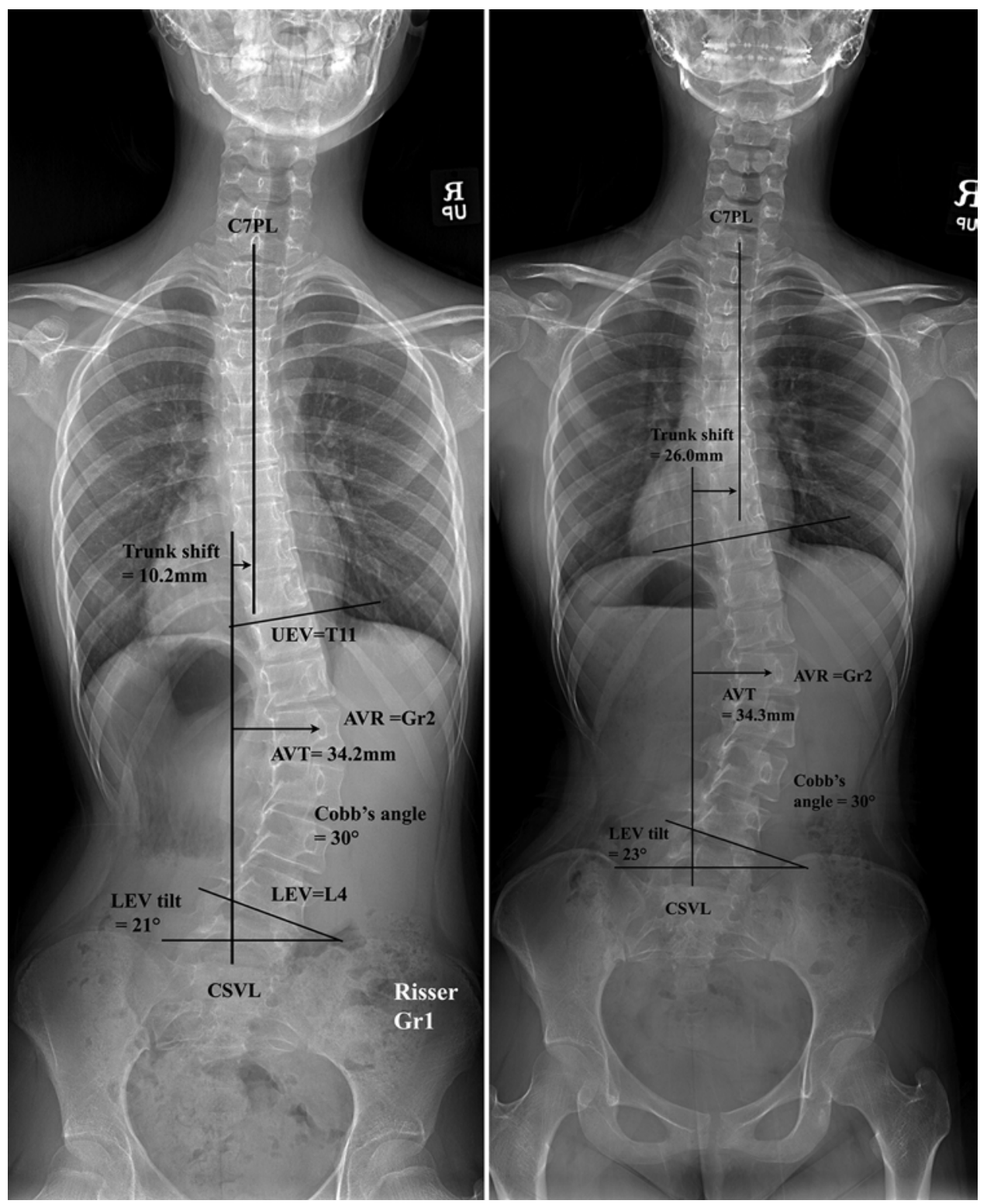

FIG. 2. Illustrative images from a 12-year-old girl with AIS and a thoracolumbar curve. Left: Standing radiograph showing trunk balance (trunk shift $=10.2 \mathrm{~mm}$ ) with Grade 2 AVR and a Grade 1 Risser sign at the initial visit. Right: Standing radiograph showing trunk imbalance (trunk shift $=26.0 \mathrm{~mm}$ ) at the 5-year follow-up. 
tween the 2 study groups, although our definition of trunk imbalance was not the same as that in previous studies, and the type of curve was different.

Interestingly, the apical vertebra is supposed to play a key role in progression of trunk imbalance in patients with a TL/L curve. In this study, logistic regression analysis identified increased rotation of the apical vertebra as being associated with the progression of trunk imbalance $(\mathrm{p}$ $=0.020$ ). These findings are supported by previous studies. One such study found that frontal decompensation can be predicted by the degree of fixed lumbar AVR after selective thoracic fusion in cases with a Lenke Type 3C curve. ${ }^{2}$ Another showed that the site of the apical vertebra is related to the direction of the curve, which may partially explain the effect of the rotational pattern of the apical vertebra on the direction of the curve, although it should be noted that this study examined cases of degenerative scoliosis. ${ }^{5}$

It has been well noted that a lower Risser grade is correlated with higher progression of curves because of higher growth potential. ${ }^{13}$ However, the relationship between skeletal age and truncal imbalance has not yet been researched. The reason for our result could be explained by the Hueter-Volkmann law, which states that bone growth is inhibited on the concave side and relatively stimulated on the convex side in skeletally immature patients. ${ }^{1}$ This was supported by the results in our study showing greater disc wedging below the LEV in Group P at the final visit $\left(5.0^{\circ}\right.$ vs $\left.2.6^{\circ}, \mathrm{p}=0.017\right)$.

The cutoff value for trunk imbalance is another controversial topic. We set the criterion for trunk imbalance as $20 \mathrm{~mm}$. However, previous studies have used $40 \mathrm{~mm}$ and $10 \mathrm{~mm}{ }^{7,15}$ Because the degree of back pain correlates with a trunk imbalance $>40 \mathrm{~mm},{ }^{7}$ the patients examined in the present study might not show any correlation between trunk imbalance and clinical symptoms.

Predicting progressive truncal imbalance is important for clinicians because of the following reasons. First, it was reported that the magnitude of the lumbar curve and trunk shift were significant predictors of surgery in TL/L AIS, ${ }^{19}$ and truncal imbalance was related to poor clinical outcomes. ${ }^{7,19}$ Second, progressive truncal imbalance could be related to pelvic asymmetrical bone growth and standing neuromuscular imbalance. ${ }^{4}$ Third, it was reported that the correction rate for trunk lateral shift was about $50 \% .{ }^{17}$ In other reports, the abnormal coronal balance was frequently observed in patients with preoperative coronal imbalance. ${ }^{9}$ So, suboptimal correction can be expected even if the surgery is performed in cases with severe truncal imbalance.

In this regard, surgeons should pay attention to progression of truncal imbalance. If patients show a lower Risser grade and greater AVR at the initial visit, then closer follow-up with radiographs should be considered. In addition, patients and their caregivers should be informed about this situation and persuaded to visit clinics more regularly with appropriate bracing. Bracing has been suggested as an effective means to prevent trunk asymmetry as well as progression of curves. ${ }^{11,20}$

This study has several limitations. First, we did not evaluate clinical outcomes such as back pain or the SRS-
22 score. We focused only on radiological predictive factors. Second, other factors such as leg length discrepancy affect trunk balance, ${ }^{7}$ as does muscle asymmetry; ${ }^{10,18}$ however, we did not evaluate leg length discrepancy or spinal muscle imbalance. Third, the sample size was too small to perform data analysis using more strict criteria. Only 13 patients $(13 / 105=12.4 \%)$ showed progressive trunk imbalance based on our criteria of a 20 -mm trunk shift at the final visit and 10-mm progression during follow-up. Thus, subgroup analysis was difficult. Nevertheless, this study is important because very few studies have tried to predict trunk imbalance, despite its clinical significance. Previous studies focused only on postoperative trunk imbalance. To the best of our knowledge, this is the first study to identify radiological factors that predict progression of trunk imbalance in those with AIS with a TL/L curve.

\section{Conclusions}

Skeletal immaturity and the AVR at the initial visit predict progression of trunk imbalance. Greater changes in LEV tilt during follow-up may also predispose patients to progressive trunk imbalance. Thus, surgeons should be aware of the risk of progressive trunk imbalance if patients show skeletal immaturity and a greater AVR at the initial visit. Closer follow-up evaluation using radiographs should be considered in this situation. However, further validation studies with larger samples are required to support these findings.

\section{Acknowledgments}

We thank Mi Young Lee, Sungjoo Cheon, So Jung Yoon, and A Lyoun Lee for help with data collection.

\section{References}

1. Arkin AM, Kate JF: The effects of pressure on epiphyseal growth: the mechanism of plasticity of growing bones. J Bone Joint Surg Am 38A:1056-1076, 1956

2. Behensky H, Cole AA, Freeman BJC, Grevitt MP, Mehdian HS, Webb JK: Fixed lumbar apical vertebral rotation predicts spinal decompensation in Lenke Type 3C adolescent idiopathic scoliosis after selective posterior thoracic correction and fusion. Eur Spine J 16:1570-1578, 2007

3. Bunnell WP: The natural history of idiopathic scoliosis before skeletal maturity. Spine (Phila Pa 1976) 11:773-776, 1986

4. Dalleau G, Leroyer P, Beaulieu M, Verkindt C, Rivard CH, Allard P: Pelvis morphology, trunk posture and standing imbalance and their relations to the Cobb angle in moderate and severe untreated AIS. PLoS One 7:e36755, 2012

5. de Vries AA, Mullender MG, Pluymakers WJ, Castelein RM, van Royen BJ: Spinal decompensation in degenerative lumbar scoliosis. Eur Spine J 19:1540-1544, 2010

6. Fortin C, Grunstein E, Labelle H, Parent S, Ehrmann Feldman D: Trunk imbalance in adolescent idiopathic scoliosis. Spine J 16:687-693, 2016

7. Glassman SD, Berven S, Bridwell K, Horton W, Dimar JR: Correlation of radiographic parameters and clinical symptoms in adult scoliosis. Spine (Phila Pa 1976) 30:682-688, 2005

8. Guo J, Liu Z, Lv F, Zhu Z, Qian B, Zhang X, et al: Pelvic tilt and trunk inclination: new predictive factors in curve progression during the Milwaukee bracing for adolescent idiopathic scoliosis. Eur Spine J 21:2050-2058, 2012 
9. Karami M, Maleki A, Mazda K: Assessment of coronal radiographic parameters of the spine in the treatment of adolescent idiopathic scoliosis. Arch Bone Jt Surg 4:376-380, 2016

10. Kim H, Lee CK, Yeom JS, Lee JH, Cho JH, Shin SI, et al: Asymmetry of the cross-sectional area of paravertebral and psoas muscle in patients with degenerative scoliosis. Eur Spine J 22:1332-1338, 2013

11. Korovessis P, Kyrkos C, Piperos G, Soucacos PN: Effects of thoracolumbosacral orthosis on spinal deformities, trunk asymmetry, and frontal lower rib cage in adolescent idiopathic scoliosis. Spine (Phila Pa 1976) 25:2064-2071, 2000

12. Li J, Hwang SW, Shi Z, Yan N, Yang C, Wang C, et al: Analysis of radiographic parameters relevant to the lowest instrumented vertebrae and postoperative coronal balance in Lenke 5C patients. Spine (Phila Pa 1976) 36:1673-1678, 2011

13. Lonstein JE, Carlson JM: The prediction of curve progression in untreated idiopathic scoliosis during growth. J Bone Joint Surg Am 66:1061-1071, 1984

14. Nash CL Jr, Moe JH: A study of vertebral rotation. J Bone Joint Surg Am 51:223-229, 1969

15. Ramirez N, Johnston CE, Browne RH: The prevalence of back pain in children who have idiopathic scoliosis. J Bone Joint Surg Am 79:364-368, 1997

16. Schwender JD, Denis F: Coronal plane imbalance in adolescent idiopathic scoliosis with left lumbar curves exceeding 40 degrees: the role of the lumbosacral hemicurve. Spine (Phila Pa 1976) 25:2358-2363, 2000

17. Seoud L, Cheriet F, Labelle H, Parent S: Changes in trunk appearance after scoliosis spinal surgery and their relation to changes in spinal measurements. Spine Deform 3:595-603, 2015

18. Shimode M, Ryouji A, Kozo N: Asymmetry of premotor time in the back muscles of adolescent idiopathic scoliosis. Spine (Phila Pa 1976) 28:2535-2539, 2003
19. Souder C, Newton PO, Shah SA, Lonner BS, Bastrom TP, Yaszay B: Factors in surgical decision making for thoracolumbar/lumbar AIS: it's about more than just the curve magnitude. J Pediatr Orthop [epub ahead of print], 2016

20. Weinstein SL, Dolan LA, Wright JG, Dobbs MB: Effects of bracing in adolescents with idiopathic scoliosis. N Engl J Med 369:1512-1521, 2013

21. Zhao Y, Wang Z, Zhu X, Wang C, He S, Li M: Prediction of postoperative trunk imbalance after posterior spinal fusion with pedicle screw fixation for adolescent idiopathic scoliosis. J Pediatr Orthop B 20:199-208, 2011

\section{Disclosures}

The authors report no conflict of interest concerning the materials or methods used in this study or the findings specified in this paper.

\section{Author Contributions}

Conception and design: Hwang. Acquisition of data: Hwang, CS Lee. Analysis and interpretation of data: Cho, DH Lee. Drafting the article: Cho. Reviewed submitted version of manuscript: Hwang, CS Lee, DH Lee. Approved the final version of the manuscript on behalf of all authors: Cho. Statistical analysis: Cho. Administrative/technical/material support: Hwang, CS Lee. Study supervision: CS Lee, DH Lee.

\section{Correspondence}

Jae Hwan Cho, Department of Orthopedic Surgery, Asan Medical Center, University of Ulsan College of Medicine, 388-1, PungNap2-dong, SongPa-gu, Seoul, Korea. email: spinecjh@gmail.com. 